\title{
Antimicrobial and cell-penetrating peptides induce lipid vesicle fusion by folding and aggregation
}

\author{
Parvesh Wadhwani · Johannes Reichert • \\ Jochen Bürck · Anne S. Ulrich
}

Received: 21 July 2011 / Revised: 6 October 2011/ Accepted: 20 October 2011/Published online: 12 November 2011 (C) The Author(s) 2011. This article is published with open access at Springerlink.com

\begin{abstract}
According to their distinct biological functions, membrane-active peptides are generally classified as antimicrobial (AMP), cell-penetrating (CPP), or fusion peptides (FP). The former two classes are known to have some structural and physicochemical similarities, but fusogenic peptides tend to have rather different features and sequences. Nevertheless, we found that many CPPs and some AMPs exhibit a pronounced fusogenic activity, as measured by a lipid mixing assay with vesicles composed of typical eukaryotic lipids. Compared to the HIV fusion peptide (FP23) as a representative standard, all designermade peptides showed much higher lipid-mixing activities (MSI-103, MAP, transportan, penetratin, Pep1). Native sequences, on the other hand, were less fusogenic (magainin 2, PGLa, gramicidin S), and pre-aggregated ones were inactive (alamethicin, SAP). The peptide structures were characterized by circular dichroism before and after interacting with the lipid vesicles. A striking correlation between the extent of conformational change and the respective fusion activities was found for the series of peptides investigated here. At the same time, the CD data show that lipid mixing can be triggered by any type of conformation acquired upon binding, whether $\alpha$-helical, $\beta$-stranded, or other. These observations suggest that lipid vesicle fusion can simply be driven by the energy released upon membrane binding, peptide folding, and possibly further aggregation. This comparative study of AMPs,
\end{abstract}

P. Wadhwani · J. Reichert · J. Bürck · A. S. Ulrich ( $\square)$ Karlsruhe Institute of Technology (KIT), Institute of Biological Interfaces (IBG-2), P.O. Box 3640, 76021 Karlsruhe, Germany e-mail: anne.ulrich@kit.edu

A. S. Ulrich

Institute of Organic Chemistry, DFG-Center for Functional Nanostructures, Fritz-Haber-Weg 6, 76131 Karlsruhe, Germany
CPPs, and FPs emphasizes the multifunctional aspects of membrane-active peptides, and it suggests that the origin of a peptide (native sequence or designer-made) may be more relevant to define its functional range than any given name.

Keywords Membrane-active peptides .

Lipid-mixing - Membrane fusion - Circular dichroism . Conformational changes · Designer-made sequences . Peptide-lipid interactions

\section{Introduction}

Membrane-active peptides exhibit a fascinating repertoire of different biological functions. Antimicrobial peptides (AMPs) can destroy bacteria by permeabilizing their lipid envelopes (Zasloff 2002), while cell-penetrating peptides (CPPs) are used to transport hydrophilic cargo across membranes without leakage (Heitz et al. 2009; Langel 2007). Fusion peptides (FPs) are generally part of larger proteins from enveloped viruses (Blumenthal et al. 2003; Jahn et al. 2003), yet they represent the minimally active agent that triggers fusion between lipid vesicles in vitro (Zheng et al. 2006). AMPs and CPPs are often cationic and amphiphilic (Almeida and Pokorny 2009), while FPs tend to be more hydrophobic and rich in small side chains such as Gly and Ala (Wilson et al. 2005). From a structural point of view, numerous AMPs and CPPs have been designed to fold into stable amphiphilic helices or $\beta$-hairpins (Henriques et al. 2006, 2007), whereas FPs are recognized by their intrinsic conformational plasticity (Reichert et al. 2007; Zheng et al. 2006). Given the similarities between many AMPs and CPPs, it is not surprising that interchangeable activities have been demonstrated for some of them, i.e., certain AMPs can penetrate membranes, and 
some CPPs are good antibiotics (Castanho 2010; Henriques et al. 2006, 2007; Luque-Ortega et al. 2008). In view of this multifunctionality, the traditional nomenclature of AMPs/ CPPs is becoming blurred, and the molecular mechanisms attributed to these "different" types of agents are not clearcut. Structural or functional similarities between AMPs/ CPPs on the one hand and FPs on the other hand are not so obvious and need to be explored. Fusion activities of AMPs/CPPs have only once been tested in the recent case of the cell-penetrating peptide TAT (Yang et al. 2010). However, all membrane-active peptides are expected to induce considerable perturbation in the lipid bilayer upon binding. Therefore, the question arises whether AMPs and CPPs may in general be able to trigger membrane fusion (Joanne et al. 2009). Here, we demonstrate by a simple lipid mixing assay that some AMPs and many CPPs are indeed strong membrane-perturbing agents. Among several available methods used to monitor vesicle fusion, lipid mixing describes the first step, leading up to at least hemifusion. We will thus take the lipid-mixing signal as an indication of membrane fusion-though not necessarily involving a well-orchestrated process.

In the literature there is no unanimous agreement on the structure of any fusogenic peptide in the membrane-bound state. A range of different conformations has been reported even for the most representative fusion peptide FP23 from HIV-1, ranging from $\alpha$-helix to $\beta$-sheet, mixtures thereof, or random coil, depending on the peptide concentration, lipid composition, and sample history (Castano and Desbat 2005; Jaroniec et al. 2005; Lorin et al. 2006; Qiang et al. 2009; Sackett et al. 2010; Sackett and Shai 2005; Zheng et al. 2006). Such conformational plasticity seems to be characteristic not only for FP23, but also for FPs in general (Lorieau et al. 2010; Sun and Weliky 2009). Nevertheless, it is important to realize that the various structural studies were performed under different conditions, and generally in the post-fusion equilibrium state. So it may not be surprising that a recent kinetic analysis of FP23 showed that the rate-limiting step of the actual fusion process involves neither an $\alpha$-helix nor a $\beta$-sheet, but rather an intrinsically flexible conformation (Reichert et al. 2007), which correlates well with a recent solid-state NMR analysis (Grasnick et al. 2011). In contrast to FPs, typical AMPs and CPPs are assumed to (or have been designed to) exhibit well-defined conformations, such as amphiphilic $\alpha$-helices. We thus selected and compared a number of exemplary peptides (Table 1) with supposedly different secondary structures, such as $\alpha$-helix, $3_{10}$-helix, polyproline type-II helix (PPII), $\beta$-turn, $\beta$-strand, disordered, and aggregated.

Ten representative AMPs and CPPs from different structural families were chosen to test their potential fusogenic action. The AMPs include the cationic magainin-2 (Tremouilhac et al. 2006) and PGLa (Afonin et al. 2008), which naturally occur in the skin of African frog Xenopus laevis. The designer-made MSI-103 is an analogue of PGLa that exhibits a higher therapeutic index (Maloy and Kari 1995; Strandberg et al. 2008). All three peptides are unstructured in aqueous solution and fold into an amphiphilic $\alpha$-helix when bound to membranes (Burck et al. 2008; Toke et al. 2004; Tremouilhac et al. 2006). The peptaibol alamethicin F30/3 from the fungus Trichoderma viride carries a single negative charge and is rather hydrophobic, with a low solubility in aqueous buffer. In membranes it folds into an $\alpha$-helix with some 310 -character, which can self-assemble to form ion channels (Maisch et al. 2009). Finally, the cationic gramicidin S from Bacillus brevis has an amphiphilic cyclic structure with $\beta$-turns (Wadhwani et al. 2006). The CPPs were selected according to their

Table 1 Investigated peptides

\begin{tabular}{|c|c|c|c|}
\hline Peptide & Type & Sequence & Proposed structure \\
\hline FP23 & FP & AVGIGALFLGFLGAAGSTMGARS-NH ${ }_{2}$ & $\alpha$-helix, $\beta$-sheet (Qiang et al. 2007; Zheng et al. 2006) \\
\hline Magainin 2 & AMP & GIGKFLHSAKKFGKAFVGEIMNS & Amphiphilic $\alpha$-helix (Maloy and Kari 1995; Tremouilhac et al. 2006) \\
\hline PGLa & AMP & GMASKAGAIAGKIAKVALKAL-NH 2 & Amphiphilic $\alpha$-helix (Afonin et al. 2008; Tremouilhac et al. 2006) \\
\hline MSI-103 & AMP & KIAGKIAKIAGKIAKIAGKIA-NH ${ }_{2}$ & Amphiphilic $\alpha$-helix (Maloy and Kari 1995; Strandberg et al. 2008) \\
\hline Alamethicin* & AMP & Ac-UPUAUAQUVUGLUPVUUEQF-ol & $\alpha$-helix, $3_{10}$-helix (Maisch et al. 2009) \\
\hline Gramicidin $S^{*}$ & AMP & Cyclo-[PVOLf PVOLf] & Cyclic with $\beta$-turns (Wadhwani et al. 2006) \\
\hline Penetratin & $\mathrm{CPP}$ & RQIKIWFQNRRMKWKK & $\alpha$-helix (Derossi et al. 1998) \\
\hline Transportan & $\mathrm{CPP}$ & $\begin{array}{l}\text { GWTLNSAGYLLGKINLKALAALAKKIL- } \\
\mathrm{NH}_{2}\end{array}$ & Kinked $\alpha$-helix (Lindberg et al. 2001) \\
\hline MAP & $\mathrm{CPP}$ & KLALKLALKALKAALKLA-NH 2 & $\alpha$-helix, $\beta$-aggregate (Scheller et al. 1999; Wadhwani et al. 2008) \\
\hline SAP & $\mathrm{CPP}$ & VRLPPPVRLPPPVRLPPP & Polyproline-II-helix (Pujals and Giralt 2008) \\
\hline Pep1 & CPP & KETWWETWWTEWSQPKKKRKV & Trp-rich (Henriques and Castanho 2004) \\
\hline
\end{tabular}

For sequences, lower case characters indicate D-amino acid; * U: AIB: aminoisobutyric acid; ol, alcoholic C-terminus; Ac, acetylated N-terminus

* O: ORN: ornithine after aminoisobutytic acid 
popular use in cellular delivery. Penetratin corresponds to a fragment from the third helix of the antennapedia homeodomain protein, but no membrane-bound structure has been reported (Derossi et al. 1998). Transportan, constructed from the $\mathrm{N}$-terminal part of the neuropeptide galanin and the wasp venom mastoparan, forms a kinked $\alpha$-helix in detergent micelles (Lindberg et al. 2001). The "model amphiphilic peptide" MAP had been designed as an analogue of the amphiphilic $\alpha$-helical AMPs described above (Scheller et al. 1999). Under many conditions, however, it aggregates into $\beta$-structures when membrane-bound (Wadhwani et al. 2008). A proline-rich analogue of maize zein, optimized for cell penetration under the name "sweet arrow peptide" SAP, forms an amphiphilic PPII-helix (Pujals and Giralt 2008). Finally, Pep1 is a Trp-rich CPP, presumably with a flexible conformation (Henriques and Castanho 2004). We note that many of these peptides, and many other CCPs in general, have some tendency to aggregate (Pujals et al. 2006).

\section{Materials and methods}

\section{Materials}

All lipids were purchased from Avanti Polar Lipids (Alabaster, AL, USA). The lipid mixture LM3 contains POPC, POPE, POPS, sphingomyelin, and cholesterol in a 10:5:2:2:10 molar ratio. Peptides were synthesized by standard Fmoc solid-phase protocols on Syro II multiple peptide synthesizer (Syro II, MultiSyntech, Germany) on a 100- $\mu$ mol scale (Fields and Noble 1990). Only alamethicin (F30) was synthesized by manual Fmoc protocols (Maisch 2008; Maisch et al. 2009). Crude peptides were purified on C18 preparative columns using a preparative RP-HPLC system (Jasco, Japan) and water-acetonitrile gradients supplemented with $5 \mathrm{mM} \mathrm{HCl}$. Purities over $95 \%$ and peptide masses were confirmed by analytical HPLC-MS ( $\mu$-TOF, Bruker, Germany) (Afonin et al. 2003; Wadhwani et al. 2006). Peptide stock solutions were prepared by weighing the dry powders in cases where there the absence of Trp in the sequence did not allow a more accurate determination by UV/VIS absorption. According to our experience with our self-synthesized/purified cationic peptides, their concentrations tend to be underestimated by up to $20 \%$ when calculated simply from the nominal molar mass of the peptide. This error can be attributed largely to the presence of counter-ions in the lyophilized material after performing HPLC with acidic solvents (here: $\mathrm{HCl}$ ). To account for this weight contribution, all amino groups were considered to be charged and accompanied by a counter-ion (here: $\mathrm{Cl}^{-}$) when calculating the effective molecular weight. Since the same stock solutions were employed for the lipid mixing assays and the CD spectroscopy, any slight error in concentration does not change the conclusions of this manuscript, i.e., that there is a correlation between lipid perturbation and peptide folding.

Preparation of large unilamellar vesicles (LUVs)

All lipids were purchased from Avanti Polar Lipids (Alabaster, AL, USA) to prepare the lipid mixture "LM-3," which is often used in viral fusion assays as it reflects the lipid head group and cholesterol composition of a typical eukaryotic target cell (Aloia et al. 1993; Yang et al. 2003). The mixture contains POPC, POPE, POPS, sphingomyelin, and cholesterol in a 10:5:2:2:10 molar ratio. Lipids and cholesterol powders were co-dissolved in chloroform, which was then removed under a gentle stream of nitrogen, followed by overnight drying under vacuum. Lipid dispersions were prepared by addition of aqueous buffers (5 mM HEPES: $\mathrm{pH} 7.0$ or $10 \mathrm{mM}$ phosphate: $\mathrm{pH} 7.0$ ) followed by vigorously vortexing $(10 \times 1 \mathrm{~min})$ and homogenization via ten freeze-thaw cycles. Finally, LUVs were prepared by extrusion through a polycarbonate filter with 100-nm diameter pores (Avanti Mini Extruder), maintaining the temperature above $40^{\circ} \mathrm{C}$ by thermostatting the extruder block at $50^{\circ} \mathrm{C}$. The formation of the vesicles was monitored by dynamic light scattering (DLS) (see below), showing that the initial multilamellar vesicles with an average diameter of about $500 \mathrm{~nm}$ and a broad size distribution yielded after 5-7 passes a constant diameter of $118 \pm 22 \mathrm{~nm}$. Therefore, 11 passes were sufficient to yield LUVs with a sharp size distribution to be used in all fusion and CD experiments. Fusion experiments were performed with vesicles containing fluorescent dyes conjugated to the lipid head groups: NBD-PE (dioleoylphosphatidylethanolamine-N-7nitro-2-1,3-benzoxadiazole-4-yl) and Rh-PE (dioleoylphosphatidylethanolamine-N-lissamine rhodamine B sulfonyl).

\section{Lipid mixing measurements}

Fusion experiments were carried out by measuring the fluorescence intensity change resulting from the fluorescence resonance energy transfer (FRET) between NBD-PE and Rh-PE inserted in the LUV bilayer (Struck et al. 1981). Vesicles containing both dyes at 2 mol\% each were mixed with dye-free vesicles at a 1:10 molar ratio to give a final lipid concentration of $150 \mu \mathrm{M}$. Fluorescence was recorded on a spectrofluorimeter (FluoroMax2, HORIBA Yobin Yvon) with excitation wavelength set at $450 \mathrm{~nm}$ and emission wavelength at $530 \mathrm{~nm}$ (6-mm slit widths). To avoid crosstalk and scattering artifacts, the emission monochromator was shielded with two cutoff filters (GG470 and GG495, $2 \mathrm{~mm}$ each, Schott, Mainz, Germany). The samples (1.5 ml) were continuously stirred, and the temperature was kept at 
$37^{\circ} \mathrm{C}$ with a thermostatic bath. The initial fluorescence of the combined vesicles was taken as $0 \%$ lipid mixing, and the extent of mixing was referred to the fluorescence level $20 \mathrm{~min}$ after addition of $2 \mathrm{~mol} \%$ peptide, measured relative to complete lipid mixing that was achieved by detergent solubilization with Triton X-100. Different peptide concentrations were tested from 1 to $7 \mathrm{~mol} \%$, resulting in a linear increase of the fluorescence signal. A concentration of $2 \mathrm{~mol} \% \mathrm{FP} 23$, corresponding to a peptide-to-lipid ratio of $1: 50$, was chosen as a suitable concentration yielding reliable signal intensity in the usual fusion reaction time of $20 \mathrm{~min}$. All peptides, except for alamethicin, were prepared as stock solutions in water $(1 \mathrm{mg} / \mathrm{ml})$ by weighing the lyophilized peptide. These were added to the vesicles to give a final peptide concentration of $3 \mu \mathrm{M}$, i.e., $2 \mathrm{~mol} \%$ relative to the amount of lipids. In the case of alamethicin the stock solution contained $50 \%$ (v/v) trifluoroethanol (TFE) in water, which resulted in an overall TFE content of 0.75 vol\% in the sample. The influence of TFE on the stability of lipid vesicles was independently examined by monitoring the fusion signals of FP23 in the presence of TFE. No significant change of the lipid mixing signals was observed up to $2 \mathrm{vol} \%$ of TFE.

The peptides Pep1, penetratin, and transportan contain Trp residues, so one has to be cautious in the data analysis because of its known quenching effect on organic dyes (Marme et al. 2003; Vaiana et al. 2003). We found that the FRET assay when performed at $530 \mathrm{~nm}$ is indeed strongly influenced in the case of Pep1 (5 Trp), for which only a very small mixing signal was detectable. For penetratin (2 Trp) the mixing signal was also slightly affected, though not for transportan (1 Trp). The ability of Pep1 to induce vesicle fusion has been previously examined in a lipid mixing assay by quantifying the (total) fluorescence decrease of Rh-PE at $590 \mathrm{~nm}$ (Henriques and Castanho 2004). At this wavelength one observes a superposition of a fast decrease (within $3 \mathrm{~s}$ ) due to Pep1 binding to the vesicles and peptide-induced quenching of rhodamine by Trp residues ( $85 \%$ of the total signal). The residual $15 \%$ of the signal consists of an additional slow decrease within the usual time scale of lipid mixing (10-15 min). Therefore, in the case of Pep1 we evaluated only the observed amplitude of the slow decrease, but at $585 \mathrm{~nm}$, and referenced this to the corresponding decrease of FP23 at the same wavelength.

\section{Vesicle size measurements}

The size of the lipid vesicles was determined before and after peptide-induced fusion by DLS (Zetasizer Nano S, Malvern Instruments Ltd.), under a scattering angle of $173^{\circ}$. Using the built-in software, the cumulant analysis yielded the mean size ( $\mathrm{z}$-average diameter) of the vesicles from the slope of the linearized form of the autocorrelation function (first cumulant). The second cumulant was used to calculate the polydispersity index (PdI), a parameter that reflects the width of the size distribution. After vesicle preparation, a z-average diameter of about $120 \mathrm{~nm}$ was observed with a PdI of $0.085 \pm 0.029$, indicating a narrow, nearly monomodal distribution, which remained unchanged for at least 3 days when stored at $4^{\circ} \mathrm{C}$. The sizes of the fused vesicles were determined $20 \mathrm{~min}$ after addition of the peptides at $3 \mu \mathrm{M}(2 \mathrm{~mol} \%)$. Aliquots $(500 \mu \mathrm{l})$ of the samples in which lipid mixing had been induced at $37^{\circ} \mathrm{C}$ were transferred to the Zetasizer instrument, and the vesicle mean size was characterized at $23^{\circ} \mathrm{C}$. However, with some peptides the fused vesicles showed a tendency to aggregate further up to some micrometers in diameter, resulting in highly fluctuating scattering events and nonanalyzable DLS data. These sticky vesicular aggregates could be readily dispersed by short sonication (15 s) in a weak ultrasonic bath, yielding stable and reproducible vesicle size determinations. The applicability of this treatment was verified with FP23, since the vesicles fused by this peptide did not change their diameter before and after more than 10 repetitive ultrasonic treatments. The peptides MSI-103, penetratin, and Pep1 required up to five sonication treatments because of the high aggregation tendency of the fused vesicles. Since the DLS-recorded diameter kept increasing over several minutes, for these peptides only the DLS values obtained within the first 1-2 min after fusion were taken as a reliable measure of the fused vesicle diameters.

\section{Circular dichroism spectroscopy}

CD spectra of the peptides were acquired on a Jasco J-810 spectropolarimeter (Jasco, Tokyo, Japan) in quartz glass cuvettes of $0.2 \mathrm{~cm}$ path length at $20^{\circ} \mathrm{C}$ in $10 \mathrm{mM}$ phosphate buffer at $\mathrm{pH} 7.0$, with and without vesicles. An average of three scans was usually recorded, and all spectra were referenced against a peptide-free solution. Stock solutions of peptides $(1 \mathrm{mg} / \mathrm{ml})$ and LUVs $(9.6 \mathrm{mM})$ were prepared in phosphate buffer, and the final peptide-to-lipid molar ratio in the cuvette was $2 \mathrm{~mol} \%$. In the case of alamethicin the peptide stock contained 50 vol\% TFE, yielding 2.5 vol\% in the CD cuvette. To maintain a constant ellipticity on the absolute scale (i.e., same number of amide bonds), the lipid-free spectra were recorded by adding $50 \mu \mathrm{l}$ of peptide solution to $450 \mu \mathrm{l}$ of phosphate buffer, resulting in a final peptide concentration of 17-45 $\mu \mathrm{M}$. In the presence of lipids, the peptide-to-lipid molar ratio was kept constant at $2 \mathrm{~mol} \%$, and the fusion reaction was allowed to be completed $\left(20 \mathrm{~min}\right.$ at $37^{\circ} \mathrm{C}$ ) before recording another set of spectra at $20^{\circ} \mathrm{C}$. To avoid absorbance flattening due to vesicle aggregation, 
Fig. 1 Fusogenic activities and structural properties of AMPs and CPPs. a Lipid-mixing activities of different AMPs (red horizontal) and CPPs (green vertical), displayed in order of decreasing activity. The amplitudes of the fluorescence resonance energy transfer (FRET) lipid-mixing signal are shown relative to the extent of fusion induced by the detergent Triton X-100 $(=100 \%)$. These values can be compared to the activity of the viral fusion peptide FP23 (left column). b Alternative assay for fusion activity based on dynamic light scattering (DLS), which gives the increase in average vesicle diameter (from originally $120 \mathrm{~nm}$ ) after peptide-induced fusion. c Circular dichroism (CD) was used to determine the extent of the conformational change experienced by the peptides upon inducing fusion. Here we show the absolute difference in mean residue ellipticity (MRE) at $195 \mathrm{~nm}$ before and after fusion (see Fig. 2). d Total charge of each peptide (including free termini). e Mean residue hydrophobicity of each peptide, normalized according to the Eisenberg consensus scale (Eisenberg et al. 1984). All experimental values (FRET, DLS, CD data) represent the mean value of three or more independent experiments

particularly at short wavelengths, the peptide/lipid spectra with MSI-103, penetratin, and Pep1 were recorded within 2 min after short (15 s) ultrasonic treatment of the samples, and five spectra were averaged.

\section{Results}

The lipid mixture LM3 reflects the lipid head group composition and cholesterol content of a typical eukaryotic plasma membrane. It is commonly used in fusion assays to mimic the response of eukaryotic cells (Aloia et al. 1993; Yang et al. 2003). Peptide-induced lipid mixing between large unilamellar LM3 vesicles was monitored by a standard FRET assay (Aloia et al. 1993; Reichert et al. 2007; Struck et al. 1981; Yang et al. 2003), and DLS served as an independent measure of vesicle size increase. To compare the AMPs and CPPs of Table 1 in a quantitative manner, each experiment was completed by a final step of detergent solubilization with Triton $\mathrm{X}-100$, setting the $100 \%$ level as complete lipid mixing (Reichert et al. 2007). This way, all values can be compared with one another and with the activity of the designated fusion peptide FP23 of HIV-1, which is used here as a reference standard.

The FRET experiments, induced by 2 mol\% peptide (i.e., a peptide-to-lipid molar ratio of 1:50), typically showed a lipid mixing signal consisting of a fast jump within 2-3 s, followed by a slow increase over 10-15 min. The jump is attributed to peptide binding, while the amplitude of the slow component is attributed to lipid mixing and was taken as a measure of the extent of vesicle fusion. The data are displayed in Fig. 1a for all peptides relative to Triton X-100, and ranked in order of decreasing activity. Remarkably, MAP, transportan, MSI-103, penetratin, and Pep1
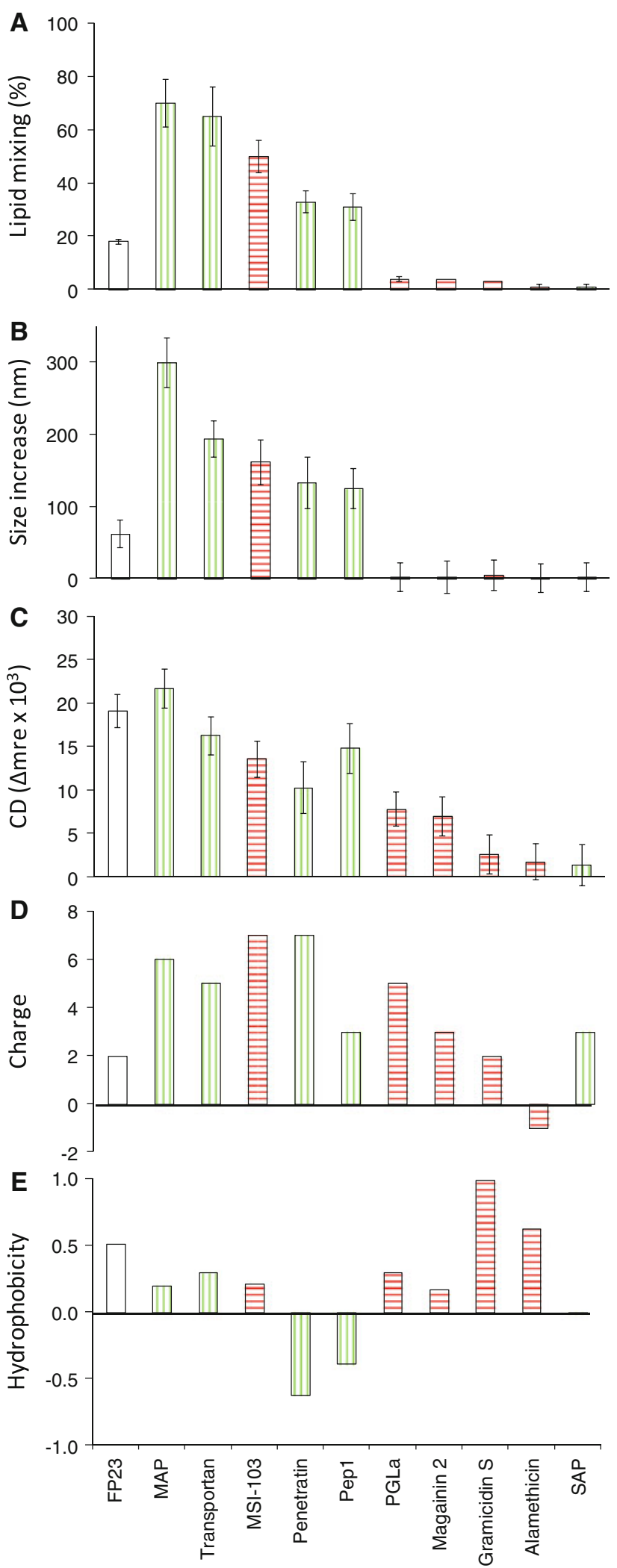

exhibit a fusion activity several times higher than FP23. The effect of the AMPs gramicidin S, magainin-2, and PGLa is about $25 \%$ compared to that of FP23, while 
alamethicin and SAP have no significant lipid-mixing activity at all.

We also monitored the vesicle size increase by DLS under the same conditions as FRET, as summarized in Fig. 1b. Starting with an average LUV diameter of about $120 \mathrm{~nm}$, the size was measured $20 \mathrm{~min}$ after addition of the peptide when the lipid mixing signal had reached a plateau. For FP23, the diameter increased from $118 \pm 24 \mathrm{~nm}$ to $180 \pm 35 \mathrm{~nm}$. The results on the AMPs and CPPs show that their ranking according to DLS follows the same order of activity that had been established by FRET. This close correspondence of vesicle size increase and lipid mixing suggests that (at least hemi-) fusion has occurred in all cases, and not just peptide-induced aggregation or lipid perturbation.
Next, all peptide systems were characterized by circular dichroism before and after the fusion process, to be able to correlate the fusion activities with any observed changes in peptide conformation. Their secondary structures were characterized by recording CD spectra, first in $10 \mathrm{mM}$ phosphate buffer at $\mathrm{pH} 7$ (dashed lines) and subsequently in the presence of the lipid vesicles after lipid mixing had been completed (solid lines), as shown in Fig. 2. These experiments were performed under the same conditions as for FRET and DLS, except that HEPES buffer was avoided because of its strong background absorbance, and the peptide concentration was scaled up to achieve a reasonable signal-to-noise ratio while maintaining a peptide-tolipid ratio of 1:50. The changes in secondary structure are summarized for all peptides in Fig. 1c by displaying the
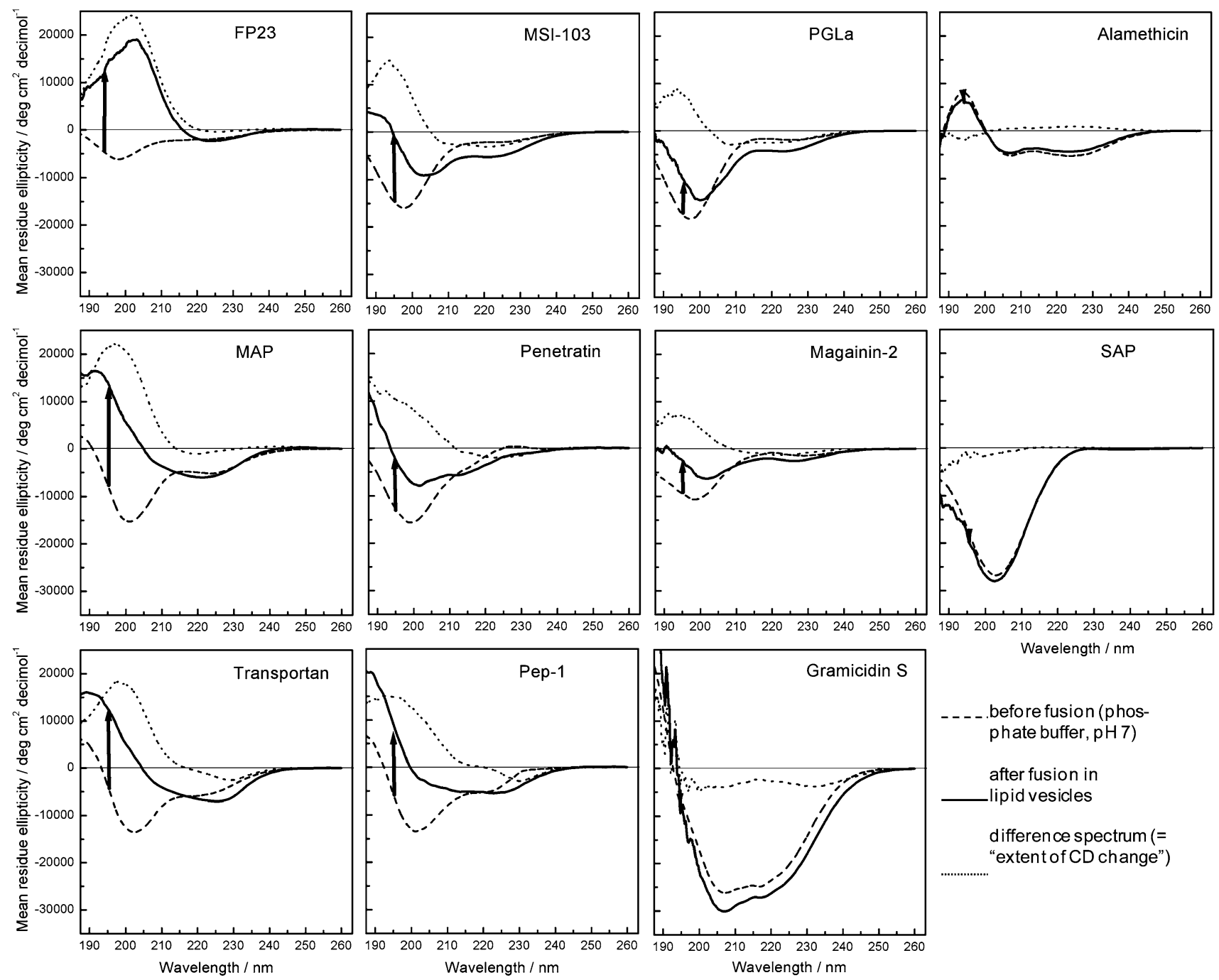

Wavelength $/ \mathrm{nm}$

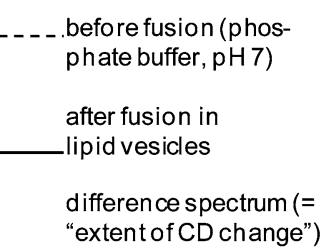

Fig. 2 Conformational change upon membrane binding. CD spectra of the peptides before fusion (dashed lines: $10 \mathrm{mM}$ phosphate buffer, $\mathrm{pH}=7$ ) and $20 \mathrm{~min}$ after inducing vesicle fusion (thick lines: LUVs of LM3 lipid mixture). The extent of the conformational change is

illustrated by the difference spectra (dotted lines) and is indicated by the arrows at $195 \mathrm{~nm}$ (where the loss of random coil signal is most pronounced) 
difference in mean residue ellipticity at $195 \mathrm{~nm}$ before and after fusion (see explanation below). Remarkably, these data are seen to essentially follow the same order of activity that had emerged from the fusion assays above.

Inspection of the $\mathrm{CD}$ lineshapes in Fig. 2 shows that most peptides have a disordered conformation in aqueous buffer, except for the sparingly soluble alamethicin and the cyclic gramicidin S. Upon fusion some of the peptides show strong conformational changes, namely FP23, MAP, and transportan (left column), which are closely followed by MSI-103, penetratin, and Pep1 (second column). PGLa, magainin-2, and gramicidin $\mathrm{S}$ exhibit weak changes (third column), and the CD spectra of alamethicin and SAP remain virtually unchanged (right column). The data show that very different types of secondary structures emerge upon interaction with the lipids, as seen from the solid lines in Fig. 2. FP23 attains a distinct $\beta$-conformation; MAP, transportan, and Pep1 exhibit resembling lineshapes with some $\beta$-stranded features, while MSI-103, penetratin, magainin, and PGLa are similar with some $\alpha$-helical features. It would be futile to describe the resulting secondary structures further or to perform a detailed spectral deconvolution. This is because it is not clear whether the peptides are $100 \%$ bound to the vesicles and assume one uniform conformation throughout the respective sample. At this point, we simply note that different types of secondary structure appear to be preferred by the different membraneactive peptides when interacting with LM3 lipid vesicles.

Despite the variations in secondary structure and possible differences in membrane affinity, we can take a look at the extent of structural change when the peptides bind to the vesicles. All peptides are essentially unstructured in aqueous buffer (except for the cyclic gramicidin $\mathrm{S}$, and the pre-aggregated alamethicin and SAP), as seen from the dashed lines in Fig. 2. It is thus justified to calculate difference spectra from the CD data from before to after fusion, as illustrated by the dotted lines in Fig. 2. These difference spectra typically exhibit a pronounced peak around $195 \mathrm{~nm}$, which reflects the loss of random coil conformation upon lipid binding, irrespective of whether any $\alpha$-helical or $\beta$-stranded conformation is gained. To express the extent of peptide folding numerically, we thus chose to evaluate the difference in CD signal intensity at $195 \mathrm{~nm}$, as illustrated by the arrows in Fig. 2. The length of the arrow in terms of CD units is the parameter displayed in Fig. 1c, beneath the lipid-mixing (Fig. 1a) and vesicle size (Fig. 1b) data. Comparing these panels, it is remarkable to see that the fusogenic activities of the diverse AMPs and CPPs correlate in a surprisingly good manner with their CD spectral changes. The only outlier in this series is the fusion peptide FP23 itself. At the same time, we can clearly show that fusion activity shows no correlation with the peptide charge or mean residue hydrophobicity (Eisenberg et al.
1984) as illustrated below in Fig. 1d and e, respectively. We thus conclude that peptide-induced vesicle fusion is accompanied by and involves considerable changes in secondary structure, and that these two aspects are directly correlated with one another.

\section{Discussion}

Membrane fusion plays a key role in many biological events, such as cell entry of enveloped viruses, fertilization, vesicle trafficking, and synaptic transmission (Blumenthal et al. 2003; Jahn et al. 2003; Ulrich et al. 1998). The fusion process is usually mediated by protein machinery that contains an apolar segment referred to as the fusion domain or fusion peptide (FP). Even as isolated fragments, these FPs are capable of inducing vesicle fusion in vitro; hence, they are widely used as model systems to study mechanistic aspects. At a molecular level, the fusion process involves a massive perturbation of the membranes, leading to a complete re-organization of the lipid molecules and merging of the two apposing bilayers. Fusion peptides naturally trigger interactions between two membranes or typically between several vesicles in vitro. An antimicrobial or cell-penetrating peptide, on the other hand, is only supposed to interact with a single lipid bilayer to fulfill its biological role of causing either local rupture (by AMPs) or transient permeation (by CPPs). Therefore, membrane fusion is not usually considered to be a relevant aspect of AMP and CPP function. From a physicochemical perspective, however, it is nonetheless clear that AMPs and CPPs also perturb the packing of the lipid molecules and may invoke a similar degree of membrane disruption. We therefore tested the ability of ten different AMPs and CPPs to induce lipid mixing among large unilamellar vesicles composed of a typical eukaryotic LM3 mixture. Figure 1a and b demonstrates that most CPPs (MAP, transportan, penetratin, Pep1), as well as the man-made antimicrobial peptide MSI-103, have potent lipid-mixing activities. Their massive fusion effects exceed even that of the reference peptide FP23. We may thus conclude that some AMPs and many CPPs are capable of triggering membrane fusion in vitro. This finding adds another complementary aspect to the previously noted multifunctionality of membrane-active peptides. For example, a typical cell-penetrating peptide like MAP or transportan can apparently also exhibit fusogenic effects as well as antimicrobial activity (Fanghänel 2011; Mink 2009). The traditional nomenclature and functional distinction between FPs and AMPs and CPPs may therefore be considered obsolete from a mechanistic point of view.

Next, the question arises as to which characteristic features are shared by the group of highly fusogenic peptides, and how they differ from the inactive ones. If a 
common denominator can be found, it might be useful for predicting the fusogenic potential of any other amino acid sequence. All peptides considered here are obviously amphiphilic and possess an intrinsic affinity to bind lipid bilayers, given their proportion of hydrophobic amino acids. However, any simple parameters such as peptide charge or mean residue hydrophobicity do not correlate with their ability to trigger fusion, as seen in Fig. 1d and e. Even some advanced concepts about membranes, such as lipid domain formation in the LM3 mixture (which should affect the ranking in terms of hydrophobic interactions) or peptide-induced charge segregation of POPS (which should affect the ranking in terms of charge), cannot therefore explain the different behavior of the peptides. We also note that it is futile to calculate the hydrophobic moment of a putative folded $\alpha$-helix (as had been done in Joanne et al. 2009), because the CD data show that this particular conformation is by no means attained by all peptides. Many of our examples display mixed folds with a significant content of $\beta$-strands, which are most likely attributed to non-specific aggregates. With this observation, it is clear that fusion activity does not correlate with the actual secondary structure of the membrane-bound peptide. Even though this question regarding a preferred "fusogenic" conformation has been raised in the past, it has led to rather conflicting observations. That is, FP23 shows $\alpha$-helical, $\beta$-stranded, or mixed $\beta$-turn conformations, depending on the lipid and sample conditions (Zheng et al. 2006). Examples have been presented where fusion was accompanied by an irreversible transition from an $\alpha$-helical structure to an antiparallel $\beta$-sheet (Castano and Desbat 2005). It has thus been proposed that an intrinsic conformational plasticity is crucial for fusion (Lorin et al. 2006). More recently, a kinetic analysis of sterically restrained FP23 analogues has suggested that the actual rate-determining step does not invoke any well-defined secondary structure at all (Reichert et al. 2007).

Our CD data show that it is not the particular type of secondary structure that matters, but it is rather the extent of the conformational change upon membrane binding that correlates with the fusion activity of a peptide. The most potent fusogenic agents (MAP, transportan) undergo the most pronounced changes in secondary structure. Peptides with a moderate conformational change are also able to trigger vesicle fusion such as MSI-103, penetratin, and Pep1, and even PGLa, magainin 2, and gramicidin S exhibit a distinct fusion activity according to FRET. Those peptides that are completely inactive, alamethicin and SAP, showed no significant change in their CD spectrum upon mixing with LM3 vesicles, as they seem to have been prestructured and pre-assembled already before encountering the vesicles. The only puzzling aspect in the otherwise excellent correlation between fusion activity and the extent of conformational change is FP23 itself. Compared to the dramatic conformational change observed, it exhibits a surprisingly low fusogenic activity and has to be regarded as an outlier. We note that even in the light of very different binding affinities of the various peptides to the LM3 vesicles, there is no question about the fact that we observe a genuine correlation here between fusion activity and conformational change. That is, consider a certain peptide that does not bind well and will simply remain in solution. This candidate will not only exhibit a correspondingly low lipid mixing activity, but at the same time it would show only a correspondingly small change in CD signal. On the other hand, a strong binder would respond vigourously in terms of both fusion and conformational change. We also note that any errors in determining the accurate peptide concentrations, which often affect the interpretation of $\mathrm{CD}$ spectra, are of no concern here either, because all experiments (FRET/DLS, and CD) were carried out using the same stock solutions. And finally, one may expect that the actual order of activities displayed in Fig. 1 is strongly affected by the choice of the particular LM3 mixture. Any other lipid composition may lead to a rather different ranking, but the correlation as such between fusion and conformational change should nevertheless be observable in the presence of different lipids.

It is important to realize that the structural properties of the representative AMPs and CPPs under investigation are not as clear-cut as may commonly be assumed (see Table 1). Only few high-resolution molecular structures are available in the relevant membrane-bound state, and on top of that the conformation in one lipid system may differ from that in another type of membrane. For example, the "model amphiphilic peptide" MAP had been designed as an ideal $\alpha$-helix as verified experimentally under several conditions in solution (Scheller et al. 1999). However, in various membranes it was found to aggregate as $\beta$-sheets in a concentration-dependent manner (Kerth et al. 2004; Wadhwani et al. 2008), as seen here also in LM3 (Fig. 2). Likewise, the cell-penetrating transportan folds as an $\alpha$-helix in detergent micelles, but a recent analysis in membranes showed distinct signs of $\beta$-sheet aggregation (Lindberg et al. 2001). In fact, it has been suggested that CPPs may generally need to undergo a certain degree of aggregation in order to fulfill their biological function (Pujals et al. 2006). Such aggregation may involve $\beta$-sheets (as in MAP and transportan), but also some other conformations have been implicated (assemblies of PPII-helices

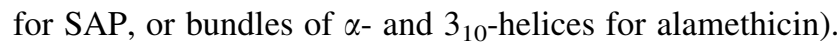
Even some AMPs such as PGLa and magainin have been demonstrated to oligomerize as dimers and/or higher oligomers, which may constitute a decisive step in their mechanism of action (Toke et al. 2004; Tremouilhac et al. 2006). Finally, for FP23 and the influenza virus fusion 
peptide, it has been demonstrated that covalent crosslinking and presumably aggregation accelerates membrane fusion (Lau et al. 2004; Yang and Weliky 2003). Therefore, it is reasonable to suppose that not only folding, but also oligomerization helps to trigger fusion, irrespective as to whether the peptide may end up as a well-defined $\alpha$-helical assembly or as an ill-defined $\beta$-sheet aggregate. The common denominator appears to be the conversion of a soluble, usually disordered form into an extensively $\mathrm{H}$-bonded state, wherein hydrophilic and hydrophobic residues are favorably positioned at the bilayer-water interface.

The driving force for membrane fusion thus appears to be the thermodynamic consequences of peptide binding, folding, and further oligomerization and/or aggregation as previously noted (Bentz and Mittal 2000, 2003; Lentz et al. 2000; Russell et al. 2001). The free energy released in this process will perturb the acyl chain packing and expose hydrophobic lipid segments to the aqueous environment, while water molecules are displaced from the membrane surface. The incoming peptides thus generate many defects and probably some negative local curvature in the bilayer such that fusion can proceed (Cevc and Richardsen 1999; Epand and Epand 2000; Lee et al. 2005). The postulated lipidic morphologies such as a stalk and transmembrane contact are neither being addressed nor questioned here (Kozlov et al. 2010). From the peptide's point of view the whole process appears to be-as we may conclude nowrather disorganized and chaotic. In the context of a wellorchestrated proteinaceous fusion apparatus, it is generally assumed that a transmembrane complex confines the site of local lipid perturbation and fusion (Blumenthal et al. 2003; Jahn et al. 2003). Once this machinery has been triggered, the fusion peptides are released and supposedly oligomerize upon contact with the opposite lipid bilayer (Qiang et al. 2009). Given the chaotic scenario described here in vitro, it is conceivable that also the process of bilayer fusion in vivo might invoke some rather disorganized local peptide structures on their way to a thermodynamically more stable post-fusion conformation.

The energetic aspects of peptide-lipid interactions generally include electrostatic attraction between peptide and lipid head groups, van der Waals interactions of hydrophobic residues with the lipid acyl chains, and the formation of H-bonds when a disordered peptide folds into a secondary structure. In our experimental set-up, electrostatics does not seem to play a prominent role, because the LM3 lipid mixture contains only about $7 \mathrm{~mol} \%$ anionic lipid, corresponding to a nominal charge of 3.5 per peptide (added to give a 1:50 peptide-to-lipid ratio). The total charge of the various AMPs and CPPs ranges from zero to +7 , but shows no direct correlation with fusion activity (Fig. 1d). Instead of charge or hydrophobicity, it is the extent of conformational change that matters. It is obvious that H-bonds are particularly favorable in the low dielectric environment of a lipid bilayer. A certain number of intramolecular H-bonds formed within an $\alpha$-helix is beneficial, and an even higher number may be achieved in a fully aggregated oligomeric $\beta$-sheet. This consideration may explain why $\beta$-stranded conformations are so often observed in membrane-bound peptides and why most of them are probably extensively aggregated to maximize the number of H-bonded contacts.

It has been suggested as a very general concept that the thermodynamically most stable state of an arbitrary protein sequence should be an aggregate, possibly with local amyloid-like $\beta$-sheet conformation (Auer et al. 2008; Chiti et al. 2003). While this postulate was derived for proteins in aqueous solution, it must apply even more so to a membraneous environment. The fact that proper protein structures exist, despite their general tendency to aggregate, has been attributed to an evolutionary selection for non-aggregating sequences. In this context, it is important to realize that every existing CPP sequence has been either rationally designed or has been cut out as a fragment from a longer protein. Therefore, it is not surprising that CPPs have not undergone any evolutionary selection against aggregation. Instead, one would expect that they have an intrinsically high propensity for aggregation and can readily convert into $\beta$-structures. The same argument also applies to fusion peptides, as these are typically cut off from larger protein machinery, by which they are kept stable only in the specific environment of their binding pocket. For antimicrobial peptides, on the other hand, the situation is completely different. Most AMPs stem from natural sources and must have been optimized by evolution not to aggregate. Only the designer-made antibiotic MSI103 has been artificially derived from PGLa. Indeed, it is found here to take on a significant $\beta$-strand conformation in LM3, and correspondingly it causes much more fusion than PGLa and magainin 2. We are therefore confident that the fundamental difference revealed here in the behavior of native peptides compared to man-made sequences should serve as a general caveat in any attempt to design pharmaceutically active peptides. Many of these products are likely to aggregate and/or misbehave in unexpected ways when encountering a lipid membrane. If this challenge is not taken into account in typical antimicrobial and cell penetration assays, a rationally optimized function might be overridden by unwanted side effects, such as peptideaggregation and possibly membrane fusion.

\section{Conclusions}

In this study we examined the ability of ten representative AMPs and CPPs to trigger membrane fusion, as monitored 
by FRET lipid-mixing and DLS vesicle size increase assays. To our surprise we found that several of these peptides had a much stronger activity than the designated fusion peptide FP23 from HIV, and this activity did not correlate with charge or hydrophobicity. Instead, CD structure analysis before and after fusion showed that those peptides that undergo a substantial conformational change upon lipid binding also turn out to be the most fusogenic. Here, the extent of change in the CD lineshape correlated directly with the fusion activity, independently of the resulting type of secondary structure ( $\alpha$-helix, $\beta$-sheet, or mixed). These observations suggest that the driving force for fusion is the energy released when a formerly disordered, soluble peptide binds to a vesicle and acquires a secondary structure by H-bond formation, which can be either a well-defined fold or an aggregated state. Pre-folded and pre-aggregated peptides, on the other hand, already exist in a stable state and are not found to promote much vesicle fusion. Regarding the origin of natural peptide sequences versus designer-made ones, it is tempting to generalize that man-made membrane-active peptides should have a high tendency to aggregate. Provided that they are water-soluble and have an affinity for membranes, we expect that they should trigger vigorous fusion of lipid vesicles.

Acknowledgments We thank Andrea Eisele, Kerstin Scheubeck, and Christian Weber for assisting in peptide synthesis and purification. Financial support from the DFG Center for Functional Nanostructures (E1.2) and the HGF-Biointerfaces Program is gratefully acknowledged.

Open Access This article is distributed under the terms of the Creative Commons Attribution Noncommercial License which permits any noncommercial use, distribution, and reproduction in any medium, provided the original author(s) and source are credited.

\section{References}

Afonin S, Glaser RW, Berditchevskaia M, Wadhwani P, Guhrs KH, Mollmann U, Perner A, Ulrich AS (2003) 4-Fluorophenylglycine as a label for F-19 NMR structure analysis of membraneassociated peptides. Chembiochem 4:1151-1163

Afonin S, Grage SL, Ieronimo M, Wadhwani P, Ulrich AS (2008) Temperature-dependent transmembrane insertion of the amphiphilic peptide PGLa in lipid bilayers observed by solid state (19)F NMR spectroscopy. J Am Chem Soc 130:16512-16514

Almeida PF, Pokorny A (2009) Mechanisms of antimicrobial, cytolytic, and cell-penetrating peptides: from kinetics to thermodynamics. Biochemistry 48:8083-8093

Aloia RC, Tian HR, Jensen FC (1993) Lipid-composition and fluidity of the human-immunodeficiency-virus envelope and host-cell plasma-membranes. Proc Natl Acad Sci USA 90:5181-5185

Auer S, Meersman F, Dobson CM, Vendruscolo M (2008) A generic mechanism of emergence of amyloid protofilaments from disordered oligomeric aggregates. PLoS Comput Biol 4:e1000222
Bentz J, Mittal A (2000) Deployment of membrane fusion protein domains during fusion. Cell Biol Int 24:819-838

Bentz J, Mittal A (2003) Architecture of the influenza hemagglutinin membrane fusion site. Biochim Biophys Acta 1614:24-35

Blumenthal R, Clague MJ, Durell SR, Epand RM (2003) Membrane fusion. Chem Rev 103:53-69

Burck J, Roth S, Wadhwani P, Afonin S, Kanithasen N, Strandberg E, Ulrich AS (2008) Conformation and membrane orientation of amphiphilic helical peptides by oriented circular dichroism. Biophys J 95:3872-3881

Castanho MARB (2010) Membrane-active peptides: methods and results on structure and function. Int Univ Line, La Jolla

Castano S, Desbat B (2005) Structure and orientation study of fusion peptide FP23 of gp41 from HIV-1 alone or inserted into various lipid membrane models (mono-, bi- and multibi-layers) by FT-IR spectroscopies and Brewster angle microscopy. Biochim Biophys Acta 1715:81-95

Cevc G, Richardsen H (1999) Lipid vesicles and membrane fusion. Adv Drug Deliv Rev 38:207-232

Chiti F, Stefani M, Taddei N, Ramponi G, Dobson CM (2003) Rationalization of the effects of mutations on peptide and protein aggregation rates. Nature 424:805-808

Derossi D, Chassaing G, Prochiantz A (1998) Trojan peptides: the penetratin system for intracellular delivery. Trends Cell Biol 8:84-87

Eisenberg D, Schwarz E, Komaromy M, Wall R (1984) Analysis of membrane and surface protein sequences with the hydrophobic moment plot. J Mol Biol 179:125-142

Epand RM, Epand RF (2000) Modulation of membrane curvature by peptides. Biopolymers 55:358-363

Fanghänel S (2011) Strukturuntersuchungen des zellpenetrierenden Peptids Transportan-10 mittels 19F-Festkörper-NMR- und orientierter Zirkulardichroismus-Spektroskopie. Institute für Organic Chemistry, vol PhD Karlsruhe Insitute of Technologie, Karlsruhe, pp 143

Fields GB, Noble RL (1990) Solid phase peptide synthesis utilizing 9-fluorenylmethoxycarbonyl amino acids. Int J Pept Protein Res 35:161-214

Grasnick D, Sternberg U, Strandberg E, Wadhwani P, Ulrich AS (2011) Irregular structure of the HIV fusion peptide in membranes demonstrated by solid-state NMR and MD simulations. Eur Biophys J 40:529-543

Heitz F, Morris MC, Divita G (2009) Twenty years of cell-penetrating peptides: from molecular mechanisms to therapeutics. Br $\mathbf{J}$ Pharmacol 157:195-206

Henriques ST, Castanho MA (2004) Consequences of nonlytic membrane perturbation to the translocation of the cell penetrating peptide pep-1 in lipidic vesicles. Biochemistry 43:9716-9724

Henriques ST, Melo MN, Castanho MA (2006) Cell-penetrating peptides and antimicrobial peptides: how different are they? Biochem J 399:1-7

Henriques ST, Melo MN, Castanho MA (2007) How to address CPP and AMP translocation? Methods to detect and quantify peptide internalization in vitro and in vivo (review). Mol Membr Biol 24:173-184

Jahn R, Lang T, Sudhof TC (2003) Membrane fusion. Cell 112:519-533

Jaroniec CP, Kaufman JD, Stahl SJ, Viard M, Blumenthal R, Wingfield PT, Bax A (2005) Structure and dynamics of micelleassociated human immunodeficiency virus gp41 fusion domain. Biochemistry 44:16167-16180

Joanne P, Nicolas P, El Amri C (2009) Antimicrobial peptides and viral fusion peptides: how different they are? Protein Pept Lett $16: 743-750$

Kerth A, Erbe A, Dathe M, Blume A (2004) Infrared reflection absorption spectroscopy of amphipathic model peptides at the air/water interface. Biophys J 86:3750-3758 
Kozlov MM, McMahon HT, Chernomordik LV (2010) Protein-driven membrane stresses in fusion and fission. Trends Biochem Sci 35:699-706

Langel Ü (2007) Handbook of cell-penetrating peptides, 2nd edn. CRC/Taylor \& Francis, Boca Raton

Lau WL, Ege DS, Lear JD, Hammer DA, DeGrado WF (2004) Oligomerization of fusogenic peptides promotes membrane fusion by enhancing membrane destabilization. Biophys J 86:272-284

Lee MT, Hung WC, Chen FY, Huang HW (2005) Many-body effect of antimicrobial peptides: on the correlation between lipid's spontaneous curvature and pore formation. Biophys J 89:4006-4016

Lentz BR, Malinin V, Haque ME, Evans K (2000) Protein machines and lipid assemblies: current views of cell membrane fusion. Curr Opin Struct Biol 10:607-615

Lindberg M, Jarvet J, Langel U, Graslund A (2001) Secondary structure and position of the cell-penetrating peptide transportan in SDS micelles as determined by NMR. Biochemistry 40:3141-3149

Lorieau JL, Louis JM, Bax A (2010) The complete influenza hemagglutinin fusion domain adopts a tight helical hairpin arrangement at the lipid: water interface. Proc Natl Acad Sci USA 107:11341-11346

Lorin A, Thomas A, Stroobant V, Brasseur R, Lins L (2006) Lipiddestabilising properties of a peptide with structural plasticity. Chem Phys Lipids 141:185-196

Luque-Ortega JR, Van't Hof W, Veerman EC, Saugar JM, Rivas L (2008) Human antimicrobial peptide histatin 5 is a cellpenetrating peptide targeting mitochondrial ATP synthesis in Leishmania. FASEB J 22:1817-1828

Maisch D (2008) Synthese und Strukturuntersuchungen des membranaktiven Peptaibols Alamethicin mittels 19F-Festkörper-NMR. Fakultät für Chemie und Biowissenschaften, vol PhD University of Kalrsruhe, Karlsruhe

Maisch D, Wadhwani P, Afonin S, Bottcher C, Koksch B, Ulrich AS (2009) Chemical labeling strategy with (R)- and (S)-trifluoromethylalanine for solid state 19F NMR analysis of peptaibols in membranes. J Am Chem Soc 131:15596-15597

Maloy WL, Kari UP (1995) Structure-activity studies on magainins and other host-defense peptides. Biopolymers 37:105-122

Marme N, Knemeyer JP, Sauer M, Wolfrum J (2003) Inter- and intramolecular fluorescence quenching of organic dyes by tryptophan. Bioconjug Chem 14:1133-1139

Mink C (2009) Zusammenhänge von Struktur und Funktion unterschiedlicher membranaktiver Peptide. Institute für Organic Chemistry, vol PhD Karlsruhe Insitute of Technologie, Karlsruhe, pp 97

Pujals S, Giralt E (2008) Proline-rich, amphipathic cell-penetrating peptides. Adv Drug Deliv Rev 60:473-484

Pujals S, Fernandez-Carneado J, Lopez-Iglesias C, Kogan MJ, Giralt E (2006) Mechanistic aspects of CPP-mediated intracellular drug delivery: relevance of CPP self-assembly. Biochim Biophys Acta 1758:264-279

Qiang W, Yang J, Weliky DP (2007) Solid-state nuclear magnetic resonance measurements of HIV fusion peptide to lipid distances reveal the intimate contact of beta strand peptide with membranes and the proximity of the Ala-14-Gly-16 region with lipid headgroups. Biochemistry 46:4997-5008

Qiang W, Sun Y, Weliky DP (2009) A strong correlation between fusogenicity and membrane insertion depth of the HIV fusion peptide. Proc Natl Acad Sci USA 106:15314-15319

Reichert J, Grasnick D, Afonin S, Buerck J, Wadhwani P, Ulrich AS (2007) A critical evaluation of the conformational requirements of fusogenic peptides in membranes. Eur Biophys J 36:405-413

Russell CJ, Jardetzky TS, Lamb RA (2001) Membrane fusion machines of paramyxoviruses: capture of intermediates of fusion. EMBO J 20:4024-4034
Sackett K, Shai Y (2005) The HIV fusion peptide adopts intermolecular parallel beta-sheet structure in membranes when stabilized by the adjacent N-terminal heptad repeat: A C-13 FTIR study. J Mol Biol 350:790-805

Sackett K, Nethercott MJ, Epand RF, Epand RM, Kindra DR, Shai Y, Weliky DP (2010) Comparative analysis of membrane-associated fusion peptide secondary structure and lipid mixing function of HIV gp41 constructs that model the early pre-hairpin intermediate and final hairpin conformations. J Mol Biol 397:301-315

Scheller A, Oehlke J, Wiesner B, Dathe M, Krause E, Beyermann M, Melzig M, Bienert M (1999) Structural requirements for cellular uptake of alpha-helical amphipathic peptides. J Pept Sci 5:185-194

Strandberg E, Kanithasen N, Tiltak D, Burck J, Wadhwani P, Zwernemann O, Ulrich AS (2008) Solid-state NMR analysis comparing the designer-made antibiotic MSI-103 with its parent peptide PGLa in lipid bilayers. Biochemistry 47:2601-2616

Struck DK, Hoekstra D, Pagano RE (1981) Use of resonance energy transfer to monitor membrane fusion. Biochemistry 20:4093-4099

Sun Y, Weliky DP (2009) 13C-13C correlation spectroscopy of membrane-associated influenza virus fusion peptide strongly supports a helix-turn-helix motif and two turn conformations. J Am Chem Soc 131:13228-13229

Toke O, O'Connor RD, Weldeghiorghis TK, Maloy WL, Glaser RW, Ulrich AS, Schaefer J (2004) Structure of (KIAGKIA)3 aggregates in phospholipid bilayers by solid-state NMR. Biophys $\mathrm{J}$ 87:675-687

Tremouilhac P, Strandberg E, Wadhwani P, Ulrich AS (2006) Synergistic transmembrane alignment of the antimicrobial heterodimer PGLa/magainin. J Biol Chem 281:32089-32094

Ulrich AS, Otter M, Glabe CG, Hoekstra D (1998) Membrane fusion is induced by a distinct peptide sequence of the sea urchin fertilization protein bindin. J Biol Chem 273:16748-16755

Vaiana AC, Neuweiler H, Schulz A, Wolfrum J, Sauer M, Smith JC (2003) Fluorescence quenching of dyes by tryptophan: interactions at atomic detail from combination of experiment and computer simulation. J Am Chem Soc 125:14564-14572

Wadhwani P, Afonin S, Ieromino M, Buerck J, Ulrich AS (2006) Optimized protocol for synthesis of cyclic gramicidin S: starting amino acid is key to high yield. J Org Chem 71:55-61

Wadhwani P, Burck J, Strandberg E, Mink C, Afonin S, Ulrich AS (2008) Using a sterically restrictive amino acid as a 19F NMR label to monitor and to control peptide aggregation in membranes. J Am Chem Soc 130:16515-16517

Wilson KA, Bar S, Maerz AL, Alizon M, Poumbourios P (2005) The conserved glycine-rich segment linking the $\mathrm{N}$-terminal fusion peptide to the coiled coil of human T-cell leukemia virus type 1 transmembrane glycoprotein gp21 is a determinant of membrane fusion function. J Virol 79:4533-4539

Yang J, Weliky DP (2003) Solid-state nuclear magnetic resonance evidence for parallel and antiparallel strand arrangements in the membrane-associated HIV-1 fusion peptide. Biochemistry 42:11879-11890

Yang R, Yang J, Weliky DP (2003) Synthesis, enhanced fusogenicity, and solid state NMR measurements of cross-linked HIV-1 fusion peptides. Biochemistry 42:3527-3535

Yang ST, Zaitseva E, Chernomordik LV, Melikov K (2010) Cellpenetrating peptide induces leaky fusion of liposomes containing late endosome-specific anionic lipid. Biophys J 99:2525-2533

Zasloff M (2002) Antimicrobial peptides of multicellular organisms. Nature 415:389-395

Zheng Z, Yang R, Bodner ML, Weliky DP (2006) Conformational flexibility and strand arrangements of the membrane-associated HIV fusion peptide trimer probed by solid-state NMR spectroscopy. Biochemistry 45:12960-12975 